\title{
A NOTE ON THE EIGENVALUES OF SADDLE POINT MATRICES
}

\section{J. LIESEN ${ }^{\dagger}$}

\begin{abstract}
Results of Benzi and Simoncini (Numer. Math. 103 (2006), pp. 173-196) on spectral properties of block $2 \times 2$ matrices are generalized to the case of a symmetric positive semidefinite block at the $(2,2)$ position. More precisely, a sufficient condition is derived when a (nonsymmetric) saddle point matrix of the form $\left[A B^{T} ;-B C\right]$ with $A=A^{T}>0$, full rank $B$, and $C=C^{T} \geq 0$, is diagonalizable and has real and positive eigenvalues.
\end{abstract}

Key words. saddle point problem, eigenvalues, Stokes problem, normal matrices

AMS subject classifications. $65 \mathrm{~F} 15,65 \mathrm{~N} 22,65 \mathrm{~F} 50$

1. Introduction. Many applications in science and engineering require solving large linear algebraic systems in saddle point form; see [1] for an extensive survey. In such problems, the system matrix often is of the form

$$
\left[\begin{array}{cc}
A & B^{T} \\
B & -C
\end{array}\right]
$$

where $A=A^{T} \in \mathbb{R}^{n \times n}$ is positive definite $(A>0), B \in \mathbb{R}^{m \times n}$ has full rank $m$, and $C=C^{T} \in \mathbb{R}^{m \times m}$ is positive semidefinite $(C \geq 0)$. The matrix in (1.1) is congruent to the block diagonal matrix $\left[\begin{array}{cc}A & 0 \\ 0 & S\end{array}\right]$, where $S=-\left(C+B A^{-1} B^{T}\right)$ with $S=S^{T}<0$. Hence the matrix in (1.1) is indefinite with $n$ positive and $m$ negative eigenvalues, which represents a significant challenge for linear solvers such as Krylov subspace methods.

It has been noted by several authors (see [1, p. 23] for references), that the matrix

$$
\mathcal{A} \equiv\left[\begin{array}{cc}
A & B^{T} \\
-B & C
\end{array}\right]
$$

which is obtained from (1.1) by multiplying the second block row by $(-1)$ is positive stable, i.e. has only eigenvalues with positive real parts; see, e.g., [1, Theorem 3.6] for a proof of this statement. What is even more appealing is that, under certain conditions, the matrix $\mathcal{A}$ is diagonalizable with all its eigenvalues real and positive. This may be advantageous when solving a linear system with $\mathcal{A}$ using a Krylov subspace method, and in addition this gives rise to a three-term recurrence conjugate gradient type method based on a positive definite inner product. The first instance of this fact has been observed by Fischer et al. [4], who considered $\mathcal{A}$ with $A=\eta I>0$, and $C=0$. Recently, the results of [4] have been extended by Benzi and Simoncini [2] to matrices $\mathcal{A}$ with $A=A^{T}>0$ and $C=0$. The purpose of this note is to generalize these results to $\mathcal{A}$ with a symmetric positive semidefinite $(2,2)$ block $C$. This is of interest in stabilized discretizations of Stokes and generalized Stokes problems; see, e.g. [3, Chapters 5-6] and [2, Section 4] for examples.

\footnotetext{
${ }^{\dagger}$ Institute of Mathematics, Technical University of Berlin, Straße des 17. Juni 136, 10623 Berlin, Germany (liesen@math.tu-berlin.de). The work of this author was supported by the Emmy Noether-Programm of the Deutsche Forschungsgemeinschaft.
} 
2. Main result. Consider a matrix $\mathcal{A}$ as in (1.2) with $A=A^{T}>0, B$ of full rank, and $C=C^{T} \geq 0$, and define the symmetric matrix

$$
\mathcal{M}_{C}(\gamma) \equiv\left[\begin{array}{cc}
A-\gamma I & B^{T} \\
B & \gamma I-C
\end{array}\right]
$$

where $\gamma$ is a yet to be specified real scalar. Note that the matrix $\mathcal{M}_{0}(\gamma)$ (i.e. $\mathcal{M}_{C}(\gamma)$ with $C=0$ ) is equal to the matrix $G$ defined in [2, p. 182]. This relation and the results for $\mathcal{M}_{0}(\gamma)$ in [2] are key ingredients in our derivation below. An elementary computation shows that

$$
\mathcal{M}_{C}(\gamma) \mathcal{A}=\mathcal{A}^{T} \mathcal{M}_{C}(\gamma)
$$

We will now derive conditions on the blocks $A, B$, and $C$ of $\mathcal{A}$ and on $\gamma$ so that $\mathcal{M}_{C}(\gamma)$ is positive definite. If these conditions are satisfied, then

$$
\mathcal{A}=\mathcal{M}_{C}(\gamma)^{-1} \mathcal{A}^{T} \mathcal{M}_{C}(\gamma)
$$

i.e., $\mathcal{A}$ is similar to its transpose by a symmetric positive definite similarity transformation. From a classical result of Taussky [8, Section 3] it then follows that $\mathcal{A}$ is similar to a real symmetric matrix. Since $\mathcal{A}$ is known to be positive real, we see that a positive definite $\mathcal{M}_{C}(\gamma)$ is a sufficient condition for $\mathcal{A}$ to be diagonalizable with all its eigenvalues real and positive.

First note that $\mathcal{M}_{C}(\gamma)$ is congruent to the block diagonal matrix

$$
\left[\begin{array}{cc}
A-\gamma I & 0 \\
0 & S
\end{array}\right], \quad \text { where } \quad S=(\gamma I-C)-B(A-\gamma I)^{-1} B^{T} .
$$

Therefore a necessary (but not sufficient) condition in order to make $\mathcal{M}_{C}(\gamma)$ positive definite is that

$$
\lambda_{\min }(A)>\gamma>\lambda_{\max }(C) .
$$

In the following we will restrict our attention to $\gamma$ satisfying (2.4). In case $A$ and $C$ are such that $\lambda_{\max }(C) \geq \lambda_{\min }(A)$, which particularly includes the case of singular $A$, the approach presented here does not work, and we are unaware of any conditions that guarantee $\mathcal{A}$ being diagonalizable with positive real eigenvalues. However, the case $\lambda_{\min }(A)>\lambda_{\max }(C)$ is of practical interest, particularly in the context of stabilized discretizations of Stokes or generalized Stokes problems. For example, the stabilized Stokes coefficient matrix in [3, p. 240] is of the form (1.1) with the $(2,2)$ block given by $-C=-\beta h^{2} D$, where $\beta$ is a nonnegative stabilization parameter and $h$ is the mesh size (here a uniform mesh is assumed for simplicity). The matrix $D$ is symmetric positive semidefinite and has norm 4 , giving $\lambda_{\max }(C)=4 \beta h^{2}$, which is is a very small number unless the stabilization parameter $\beta$ is chosen very large. In particular, for any symmetric positive definite $A, \lambda_{\min }(A)>\lambda_{\max }(C)$ holds for all $\beta<\frac{1}{4} h^{-2} \lambda_{\min }(A)$.

Next, using a standard result on the eigenvalues of symmetric matrices (cf. e.g. $[5$, Theorem 8.1.5]),

$$
\begin{aligned}
\lambda_{\min }\left(\mathcal{M}_{C}(\gamma)\right) & \geq \lambda_{\min }\left(\left[\begin{array}{cc}
A-\gamma I & B^{T} \\
B & \gamma I
\end{array}\right]\right)+\lambda_{\min }\left(\left[\begin{array}{cc}
0 & 0 \\
0 & -C
\end{array}\right]\right) \\
& =\lambda_{\min }\left(\mathcal{M}_{0}(\gamma)\right)-\lambda_{\max }(C)
\end{aligned}
$$


Hence a sufficient condition so that $\mathcal{M}_{C}(\gamma)$ is positive definite is

$$
\lambda_{\min }\left(\mathcal{M}_{0}(\gamma)\right)>\lambda_{\max }(C) .
$$

To derive properties on $A, B, C$, and $\gamma$ so that (2.6) holds, we consider the eigenvalue problem $\mathcal{M}_{0}(\gamma)\left[x^{T} ; y^{T}\right]^{T}=\theta\left[x^{T} ; y^{T}\right]^{T}$, or

$$
\text { (i) }(A-\gamma I) x+B^{T} y=\theta x \text {, and } \quad(i i) \quad B x+\gamma y=\theta y \text {. }
$$

If there exists an eigenvalue $\theta$ with $\theta=\gamma$, then $\theta=\gamma>\lambda_{\max }(C)$ since we have restricted our attention to $\gamma$ satisfying (2.4). If $\theta \neq \gamma$ we can transform equation ( $i i)$ into its equivalent form $y=(\theta-\gamma)^{-1} B x$, which, inserted into $(i)$ yields

$$
(A-\gamma I) x+(\theta-\gamma)^{-1} B^{T} B x=\theta x .
$$

Note that we must have $x \neq 0$ for if otherwise equation $(i i)$ would yield $y=0$, a contradiction to the fact that $\left[x^{T}, y^{T}\right]^{T}$ is an eigenvector. After multiplying from the left with $x^{T}$ and some algebraic manipulations we obtain the equation

$$
\theta+\gamma^{2} \frac{x^{T} x}{x^{T} A x}=\theta^{2} \frac{x^{T} x}{x^{T} A x}+\gamma-\frac{x^{T} B^{T} B x}{x^{T} A x}
$$

As in the proof of [2, Corollary 3.2], we can bound the left hand side of (2.7) from above by $\theta+\gamma^{2} / \lambda_{\min }(A)$, and the right hand side from below by

$$
\gamma-\frac{x^{T} B^{T} B x}{x^{T} A x} \geq \gamma-\lambda_{\max }\left(B A^{-1} B^{T}\right),
$$

which yields the following lower bound on $\theta$,

$$
\theta \geq \gamma-\frac{\gamma^{2}}{\lambda_{\min }(A)}-\lambda_{\max }\left(B A^{-1} B^{T}\right)
$$

To maximize the lower bound on $\theta$ we set $\gamma=\gamma^{*} \equiv \frac{1}{2} \lambda_{\min }(A)$. This value of $\gamma$ is also used in [2], and it is there determined by a slightly different argument in the proof of Proposition 3.1. With $\gamma=\gamma^{*}$, (2.8) becomes

$$
\theta \geq \frac{1}{4} \lambda_{\min }(A)-\lambda_{\max }\left(B A^{-1} B^{T}\right)
$$

Combining this with (2.6) shows that $\mathcal{M}_{C}\left(\gamma^{*}\right)$ is positive definite when

$$
\lambda_{\min }(A)>4\left(\lambda_{\max }(C)+\lambda_{\max }\left(B A^{-1} B^{T}\right)\right) .
$$

Note that if $(2.10)$ holds, and $\gamma=\gamma^{*}$, then the necessary condition (2.4) on $\gamma$ is satisfied. We summarize our discussion in the following theorem.

Proposition 2.1. Consider the matrix $\mathcal{A}$ as in (1.2) with symmetric positive definite $A \in \mathbb{R}^{n \times n}, B \in \mathbb{R}^{m \times n}$ of full rank $m$, and symmetric positive semidefinite $C \in \mathbb{R}^{m \times m}$, and let $\gamma^{*} \equiv \frac{1}{2} \lambda_{\min }(A)$. If (2.10) holds, then the matrix $\mathcal{M}_{C}\left(\gamma^{*}\right)$ in (2.1) is positive definite, and $\mathcal{A}$ is diagonalizable with all its eigenvalues real and positive.

This proposition is a generalization of results previously obtained in $[4,2]$ :

Fischer et al. [4] consider $\mathcal{A}$ with $A=\eta I>0$ and $C=0$. The condition (2.10) then reads $\eta>2 \sigma_{\max }(B)$, where $\sigma_{\max }(B)$ denotes the largest singular value of $B$. 
This is precisely the condition derived in [4, pp. 531-532], and the matrix $\mathcal{M}_{0}(\eta / 2)$ in (2.1) is equal to the matrix in [4, Equation (2.3)] multiplied by $\eta / 2$.

Benzi and Simoncini [2, Section 3] consider $\mathcal{A}$ with $A=A^{T}>0$ and $C=0$. Their matrix $G$ in [2, p. 182] is equal to $\mathcal{M}_{0}(\gamma)$ in $(2.1)$, and [2, Proposition 3.1] is equivalent with Proposition 2.1 above. For the case $C=\beta I \geq 0,[2$, Corollary 2.6] shows that if $\lambda_{\min }(A) \geq 3 \beta+4 \lambda_{\max }\left(B A^{-1} B^{T}\right)$, then $\mathcal{A}$ has real eigenvalues. The condition on $\beta=\lambda_{\max }(C)$ in this special case is a bit weaker than (2.10). Note however that (2.10) not only implies real eigenvalues but also diagonalizability of $\mathcal{A}$.

In the terminiology of [6] and under the condition (2.10), the matrix $\mathcal{A}$ is normal of degree one with respect to the symmetric positive definite matrix $\mathcal{M}_{C}\left(\gamma^{*}\right)$. According to $[6$, Theorem 3.1], $\mathcal{A}$ must be diagonalizable. If we write the eigendecomposition as $\mathcal{A}=W \Lambda W^{-1}$, where the eigenvalues and eigenvectors of $\mathcal{A}$ are ordered so that the same eigenvalues form a single block on the diagonal of $\Lambda$, then $\mathcal{M}_{C}\left(\gamma^{*}\right)$ must be of the form $\mathcal{M}_{C}\left(\gamma^{*}\right)=\left(W D W^{T}\right)^{-1}$, where $D$ is a symmetric positive definite block diagonal matrix with block sizes corresponding to those of $\Lambda$, cf. [6, Theorem 3.1]. With $\hat{W}=W D^{-1 / 2}, \mathcal{M}_{C}\left(\gamma^{*}\right)=\left(\hat{W} \hat{W}^{T}\right)^{-1}$, and thus

$$
\kappa\left(\mathcal{M}_{C}\left(\gamma^{*}\right)\right)=\left\|\mathcal{M}_{C}\left(\gamma^{*}\right)\right\|\left\|\mathcal{M}_{C}\left(\gamma^{*}\right)^{-1}\right\|=\kappa(\hat{W})^{2}
$$

(cf. [2, pp. 184-185], where a similar result is derived in a different way, and subsequently used to bound the residual norm of a Krylov subspace method applied to the matrix $\mathcal{A}$ ). An estimate for these quantities can be found as follows: First, by [5, Theorem 8.1.5] and [2, Corollary 3.2],

$$
\lambda_{\max }\left(\mathcal{M}_{C}\left(\gamma^{*}\right)\right) \leq \lambda_{\max }\left(\mathcal{M}_{0}(\gamma)\right) \approx \lambda_{\max }(A),
$$

and second, by (2.5) and (2.9),

$$
\lambda_{\min }\left(\mathcal{M}_{C}\left(\gamma^{*}\right)\right) \geq \frac{1}{2} \gamma^{*}-\left(\lambda_{\max }(C)+\lambda_{\max }\left(B A^{-1} B^{T}\right)\right) .
$$

Combining these two inequalities yields

$$
\kappa\left(\mathcal{M}_{C}\left(\gamma^{*}\right)\right)=\frac{\lambda_{\max }\left(\mathcal{M}_{C}\left(\gamma^{*}\right)\right)}{\lambda_{\min }\left(\mathcal{M}_{C}\left(\gamma^{*}\right)\right)} \approx \frac{\lambda_{\max }(A)}{\frac{1}{2} \gamma^{*}-\left(\lambda_{\max }(C)+\lambda_{\max }\left(B A^{-1} B^{T}\right)\right)} .
$$

For $C=0$ this result corresponds to the one given in [2, Corollary 3.2].

Since $\mathcal{A}$ is normal of degree one with respect to $\mathcal{M}_{C}\left(\gamma^{*}\right), \mathcal{A}$ admits an optimal three-term recurrence for computing Krylov subspace bases that are orthogonal with respect to the inner product generated by $\mathcal{M}_{C}\left(\gamma^{*}\right),\langle x, y\rangle \equiv y^{T} \mathcal{M}_{C}\left(\gamma^{*}\right) x$; see [6] for details. Therefore, a three-term recurrence conjugate gradient type method based on this inner product can be constructed. For a practical application of such method a preconditioner that is symmetric positive definite with respect to this inner product should be available, and the inner product matrix $\mathcal{M}_{C}\left(\gamma^{*}\right)$ should be well conditioned. While the condition number of $\mathcal{M}_{C}\left(\gamma^{*}\right)$ depends on the conditioning of the eigenvectors of $\mathcal{A}$ and can be estimated as shown above, the construction of such preconditioners is an open problem.

Finally, as a simple example we consider the matrix

$$
\mathcal{A}=\left[\begin{array}{ccc|cc}
1 & 0 & 0 & b & 0 \\
0 & 2 & 0 & 0 & b \\
0 & 0 & 3 & 0 & 0 \\
\hline-b & 0 & 0 & 2 c & -c \\
0 & -b & 0 & -c & 2 c
\end{array}\right], \quad b \neq 0, \quad c \geq 0
$$


Elementary computations show that

$$
\lambda_{\min }(A)=1, \quad \lambda_{\max }\left(B A^{-1} B^{T}\right)=b^{2}, \quad \lambda_{\max }(C)=3 c,
$$

and hence the sufficient condition (2.10) becomes

$$
1>12 c+4 b^{2} \text {. }
$$

If we choose $b=1 / 2$, then this condition is not satisfied for any $c \geq 0$, and indeed a MATLAB [7] computation reveals that the matrix $\mathcal{A}$ is not diagonalizable for $c=0$, and has eigenvalues with nonzero imaginary parts for $c>0$. On the other hand, if we choose $c=1 / 12$, then a MATLAB computation shows that $\mathcal{A}$ has five distinct real and positive eigenvalues whenever $|b| \leq 0.4056855$.

Acknowledgements. Part of this work was done during my visit of Emory University in April 2006. I thank Michele Benzi for his kind hospitality and for very helpful discussions and suggestions. I also thank Valeria Simoncini and Petr Tichý for their comments.

\section{REFERENCES}

[1] M. Benzi, G. H. Golub, And J. Liesen, Numerical solution of saddle point problems, Acta Numer., 14 (2005), pp. 1-137.

[2] M. Benzi ANd V. Simoncini, On the eigenvalues of a class of saddle point matrices, Numer. Math., 103 (2006), pp. 173-196.

[3] H. C. Elman, D. J. Silvester, And A. J. Wathen, Finite elements and fast iterative solvers: with applications in incompressible fluid dynamics, Numerical Mathematics and Scientific Computation, Oxford University Press, New York, 2005.

[4] B. Fischer, A. Ramage, D. J. Silvester, and A. J. Wathen, Minimum residual methods for augmented systems, BIT, 38 (1998), pp. 527-543.

[5] G. H. Golub and C. F. Van Loan, Matrix computations, Johns Hopkins Studies in the Mathematical Sciences, Johns Hopkins University Press, Baltimore, MD, third ed., 1996.

[6] J. LiESEn AND Z. StRAKoš, On optimal short-term recurrences for generating orthogonal Krylov subspace bases, in preparation, (2006).

[7] MATLAB, The MathWorks Company, Natick, MA. http://www.mathworks.com.

[8] O. TAussky, The role of symmetric matrices in the study of general matrices, Linear Algebra and Appl., 5 (1972), pp. 147-154. 